\title{
Tratamiento de los sarcomas estromales del tracto gastrointestinal: papel del imatinib (Glivec)
}

\author{
J. M. Buesa, J. Fra
}

\section{Introducción}

Los sarcomas del estroma gastrointestinal (GIST) se originan normalmente en la pared gastrointestinal (esófago, estómago o intestino), pero también pueden surgir en el mesenterio, el epiplón, el retroperitoneo o en la pared abdominal. En ausencia de síntomas o de sangrado, su diagnóstico es tardío y los enfermos suelen presentarse con enfermedad avanzada. Las recaídas o las metástasis se localizan en el peritoneo o el hígado, rara vez a distancia (pulmón o hueso), y el fallecimiento del paciente ocurre por complicaciones relacionadas con la extensión abdominal del tumor. El tratamiento es quirúrgico, aunque diversos aspectos del manejo del GIST se están modificando desde la introducción, en el año 2000, del medicamento imatinib (Glivec).

\section{Cirugía}

El tratamiento de elección es la extirpación en bloque con márgenes microscópicos negativos (R0), y las resecciones muy extensas no han mostrado ventajas sobre las resecciones en cuña. En ocasiones, puede ser necesario sacrificar órganos adyacentes para lograr la exéresis completa del tumor, pero no sería preciso extirpar estructuras vitales para conseguir unos márgenes adecuados cuando no se deja tumor macroscópico. La rotura del tumor (R1), antes o durante la intervención entraña mal pronóstico, por lo que es necesario disecar cuidadosamente el tumor; si esto ocurre, es recomendable el lavado de la cavidad peritoneal para eliminar la posible presencia de células tumorales. La biopsia percutánea comporta un riesgo de rotura del tumor o de sangrado, y debería evitarse. Estos tumores rara vez afectan a los ganglios, por lo que no está indicada una linfadenectomía sistemática. Debe intentarse la resección de las metástasis, bien en el acto quirúrgico inicial, bien después de un trata-

Servicio de Oncología Médica Hospital General de Asturias Oviedo miento con imatinib (Glivec), como veremos más adelante. La radioterapia o la quimioterapia convencionales después de la cirugía no tienen una eficacia probada ${ }^{1,2}$.

\section{Resultados de la cirugía}

La supervivencia a los 5 años observada en distintas series quirúrgicas, que incluyen pacientes con enfermedad tanto localizada como metastásica, oscila entre el 35 y el $65 \%$, y la recaída ocurre en el $60 \%$ de los casos en los 2 primeros años de seguimiento ${ }^{1,2}$. En una serie de 80 pacientes con un GIST no metastásico resecado por completo, el $40 \%$ de los pacientes presentó una recidiva después de un seguimiento medio de 24 meses, y el $46 \%$ había fallecido por el tumor a los 5 años ${ }^{2}$.

El rescate quirúrgico de las recaídas o de las metástasis es poco satisfactorio, y estaría indicado para alivio sintomático. Puede lograrse en un 33\% de los casos, pero la supervivencia media ulterior es de unos 15 meses, señalando la presencia de enfermedad oculta diseminada, que da lugar a nuevas recidivas ${ }^{3}$. Los tratamientos de quimioterapia clásica disponibles no son efectivos frente al GIST, y las escasas series publicadas de enfermos tratados con radioterapia han arrojado resultados negativos.

\section{Factores pronóstico}

Se han combinado el tamaño del tumor primario y el índice mitótico (por 50 campos de gran aumento), para clasificar a los GIST en 4 grupos de riesgo de recaída: riesgo muy bajo ( $<2 \mathrm{~cm}$ y $<5$ mitosis), riesgo bajo ( $2-5 \mathrm{~cm}$ y $<5$ mitosis), riesgo intermedio $(<5 \mathrm{~cm}$ y $6-10$ mitosis; o $5-10 \mathrm{~cm}$ y $<5 \mathrm{mi}-$ tosis) y riesgo alto ( $>5 \mathrm{~cm}$ y $>5$ mitosis; $>10 \mathrm{~cm}$ o $>10$ mitosis) (4). La supervivencia a los 5 años de los 3 primeros grupos oscila entre el 70 y el $85 \%$, mientras que el $68 \%$ de los pacientes de riesgo alto fallece a causa de su tumor, el $50 \%$ en los 2,5 años siguientes al diagnóstico ${ }^{5}$.

\section{Tratamiento con mesilato de imatinib (Glivec)}

El $90 \%$ de los GIST presentan mutaciones del gen c-kit que determinan la activación permanente de la tirosínquina- 
sa del receptor c-kit. Se activan así distintas vías de señales que determinan el comportamiento maligno de estos tumores. El mesilato de imatinib (Glivec) es una fenilaminopirimidina que se desarrolló para inhibir la función de la tirosínquinasa de la proteína de fusión Bcr-Abl, asociada con la leucosis mieloide crónica. Posteriormente se comprobó que inhibía la actividad de la tirosínquinasa de c-kit y del receptor del factor de crecimiento derivado de las plaquetas (PDGFR). Este fármaco se une al dominio intracelular de la quinasa responsable de interaccionar con el adenosíntrifosfato, interrumpiendo la cascada de señales. Después de comprobar su eficacia frente a líneas celulares de GIST con mutaciones activantes de c-kit, se comprobó la eficacia del fármaco en un paciente con un GIST avanzado. En biopsias seriadas se ha observado que el tratamiento causa una fibrosis y una degeneración mixoide del tumor, que conserva su arquitectura vascular ${ }^{6,7}$.

Los resultados de los ensayos clínicos en pacientes con un GIST avanzado c-kit positivo (determinado por técnicas de inmunohistoquímica), iniciados en el año 2000, han supuesto un cambio radical en el pronóstico de estos enfermos, dado que el tumor es resistente a los citotóxicos habituales. Un ensayo clínico incluyó 27 pacientes tratados con imatinib $400 \mathrm{mg} / 12$ horas. Sólo 3 (11\%) progresaron de entrada al tratamiento, y el $73 \%$ no había progresado al cabo de un año ${ }^{8}$. Otro estudio, que incluyó 147 pacientes, comparó una dosis de 400 con una de 600 mg/día, sin encontrar diferencias en actividad ni en toxicidad?. En una actualización de estos resultados, el $67 \%$ de los pacientes mostró una respuesta objetiva, el 17\% estabilización y sólo el 10\% progresó. El tiempo medio hasta alcanzar la respuesta fue de 13 semanas, y el tiempo medio a la progresión 29 meses, de forma que el $83 \%$ de los pacientes estaba vivo después de un seguimiento actuarial de 21 meses. La respuesta al tratamiento estaba influida por la localización de la mutación en c-kit (exón 11 ○ 9), pero incluso el 30\% de pacientes sin mutación en estos exones puede presentar una estabilización prolongada $^{10}$. Dos estudios han comparado la dosis de 400 con la de $800 \mathrm{mg} /$ día. Los resultados provisionales no muestran diferencias ni en actividad ni en toxicidad, por lo que la dosis recomendada de imatinib es de $400 \mathrm{mg}$ /día de forma ininterrumpida.

El imatinib (Glivec) se absorbe bien por vía oral y una dosis de $400 \mathrm{mg}$ diarios proporciona unas concentraciones plasmáticas por encima de las necesarias para inhibir el receptor c-kit (6). El fármaco es bien tolerado y sólo el $25 \%$ de los pacientes tratados presenta algún efecto adverso importante (grado $3 \circ 4$ ), cuya intensidad no parece relacionada con la dosis. Los efectos secundarios más frecuentes son anemia (92\%), edema (especialmente periorbitario) $(84 \%)$, erupción cutánea $(69 \%)$, cansancio $(76 \%)$, náuseas $(57 \%)$, neutropenia (47\%) o diarrea (47\%). Algunos enfermos pueden presentar una hemorragia relacionada con la necrosis tumoral provocada por el fármaco y deben ser intervenidos para resolver el problema. La larga supervivencia esperable con el tratamiento justifica una actitud agresiva ante ésta $u$ otra complicación que pueda surgir ${ }^{8,10,11}$.

La captación tumoral de ${ }^{18} \mathrm{~F}$-deoxiglucosa, medida mediante la tomografía de emisión de positrones (PET), puede estar ya reducida a las 24 horas de iniciarse el imatinib en los pacientes que responden al tratamiento. Dado que la estuctura del tumor se conserva, la PET es superior a la tomo- grafía computarizada (TC) para detectar la eficacia o ineficacia del fármaco. Además, los cambios en la TC son tardíos y las lesiones pueden aumentar de tamaño (edema relacionado con la respuesta) o cambiar de densidad, siendo difícil aplicar los criterios de respuesta habituales, basados en la medida de las lesiones. Como la PET no está disponible en muchos centros, se han comparado la PET y la TC en esta situación clínica, y quizá los criterios de respuesta del GIST evaluado mediante una TC deberían revisarse ${ }^{12}$.

Hay muchos aspectos todavía mal conocidos en relación con la evolución de los GIST tratados con imatinib. La idea general es que, aunque el imatinib consigue un control prolongado de la enfermedad metastásica, el tumor finalmente se hace resistente, la enfermedad progresa y el paciente fallece a causa del tumor. Por este motivo debe intentarse la resección del tumor cuando se observe mejoría con imatinib (especialmente con la PET), y se está acumulando una experiencia favorable, todavía limitada, con este planteamiento ${ }^{13}$. Además, se está estudiando la eficacia del imatinib en pacientes sometidos a una resección completa del tumor y se están desarrollando otros fármacos semejantes, que puedan ser eficaces en pacientes con un GIST resistente a imatinib.

\section{Tratamiento complementario}

Se han diseñado 3 estudios prospectivos y aleatorizados para evaluar el impacto que el imatinib pueda tener en la evolución de los pacientes sometidos a una resección completa de un GIST. El planteamiento es muy semejante: la mitad de los enfermos recibe $400 \mathrm{mg}$ de imatinib por 1-2 años y la otra mitad es sometida a controles periódicos, aunque los estudios difieren en los criterios de selección. Nuestro centro, integrado en el Grupo Español de Investigación en Sarcomas (GEIS), tiene previsto participar en el ensayo EORTC 64024. Son elegibles para este estudio pacientes con un riesgo alto o intermedio, sometidos a una exéresis completa del tumor. Se valorará la supervivencia libre de enfermedad y la supervivencia global. Se pretende detectar una mejora del $15 \%$ (pasar del 50 al $65 \%$ ) en la supervivencia a los 5 años, para lo que se requiere una muestra de 400 pacientes. A estos ensayos clínicos se asocia el análisis molecular del tejido tumoral, antes y después del tratamiento (en caso de recaída), con objeto de establecer correlaciones entre las alteraciones moleculares iniciales, su posible variación en el tiempo y la evolución de la enfermedad.

\section{Bibliografía}

1. Ng EH, Pollock RE, Romsdahl MM. Prognostic implications of patterns of failure for gastrointestinal leiomyosarcomas. Cancer 1992; 69:1334-41

2. DeMatteo RP, Lewis JJ, Leung D, et al. Two hundred gastrointestinal stroma tumors. Recurrence patterns and prognostic factors for survival. Ann Surg 2000; 231:51-8.

3. Mudan SS, Conton KC, Woodruff JM, et al. Salvage surgery for patients with recurrent gastrointestinal sarcoma. Prognostic factors to guide patient selection. Cancer2000; 88: 66-74.

4. Fletcher $C$, Berman JJ, Corless $C$, et al. Diagnosis of gastrointestinal stromal tumors: a consensus approach. Hum Pathol 2002; 33:459-65 


\section{J. M. Buesa y J. Fra}

5. Kindblom LG. Gastrointestinal stromal tumors. Diagnosis, epidemiology, prognosis. A.S.C.O. Virtual Meeting 2003. Disponible en www.asco.org

6. Manley PW, Cowan-Jacob SW, Buchdunger E, et al. Imatinib: a selective tyrosine kinase inhibitor. Eur J Cancer 2002; 38 (Suppl 5): S19-S27.

7. Demetri GD. Identification and treatment of chemoresistant inoperable or metastatic GIST: experience with the selective tyrosine kinase inhibitor imatinib mesylate (STI571). Eur J Cancer 2002; 38 (Suppl 5): S52-S59.

8. Verweij J, van Oosterom A, Blay JY, et al. Imatinib mesylate is an active agent for gastrointestinal stromal tumours, but does not yield responses in other soft-tissue sarcomas that are unselected for a molecular target. Eur J Cancer 2003; 39: 2006-11.

9. Demetri GD, von Mehren M, Blanke Ch, et al. Efficacy and sa- fety of imatinib mesylate in advanced gastrointestinal stromal tumors. N Eng J Med 2002; 347: 472-80.

10. Blanke Ch. Long-term follow up of advanced gastrointestinal stromal (GIST) patients treated with imatinib mesylate. 2004 Gastrointestinal Cancers Symposium. Disponible en: www.asco.org

11. Croom KF y Perry CM. Imatinib mesylate. Treatment of gastrointestinal stromal tumors. Drugs 2003; 63:513-22.

12. Gayed I, Vu T, lyer $R$, et al. The role of 18F-FDG PET in staging and early detection of response to therapy of recurrent gastrointestinal stromal tumors. J Nucl Med 2004; 45:17-21.

13. Bümming $P$, Andersson J, Meis-Kindblom JM, y cols. Neoadjuvant, adjuvant and palliative treatment of gastrointestinal stromal tumors (GIST) with imatinib: a centre-based study. $\mathrm{Br} \mathrm{J}$ Cancer 2003; 89: 460-4. 